\title{
Efectos de la certificación Florverde Sustainable Flowers en las empresas floricultoras afiliadas a Asocolflores*
}

\section{Effects of the Florverde Sustainable Flowers certification on floriculture companies affiliated with Asocolflores}

\section{RESUMEN}

Este estudio tuvo como objetivo determinar las diferencias entre las empresas floricultoras certificadas con el esquema Florverde Sustainable Flowers (FSF) y las que no lo están, con respecto a sus prácticas sociales y ambientales en el marco de la responsabilidad social (RS). Con base en las prácticas de RS incluidas en FSF se aplica una encuesta de opinión a los directivos y se realizan entrevistas a los trabajadores. La identificación de diferencias entre las empresas certificadas y no certificadas permite establecer los efectos de la certificación FSF en las empresas estudiadas. Se encuentran diferencias en la implementación de prácticas de RS para 41 de las 74 variables analizadas en las categorías de medio ambiente, prácticas laborales y asuntos de consumidores. Los beneficios de la certificación FSF se analizan desde dos perspectivas; desde la perspectiva interna, FSF contribuye a la producción de flores con un enfoque sostenible, a organizar los procesos internos y a cumplir con la legislación aplicable. Desde la perspectiva externa, FSF mejora la imagen de las

Artículo de resultado de investigación.

** Ingeniero agrónomo. Especialista en Mercadeo. Magíster en Calidad y Gestión Integral. Profesional senior de certificación en el Instituto Colombiano de Normas Técnicas y Certificación. Correo electrónico: ricardobogotar@gmail.com 
empresas ante el mercado internacional, los trabajadores y la comunidad. Las empresas del sector tienen en el esquema FSF una valiosa herramienta para aplicar las materias fundamentales y los asuntos de la RS en sus operaciones.

Palabras clave: Florverde, flores, responsabilidad social, sostenibilidad, certificación socioambiental.

\section{ABSTRACT}

This study aimed to determine if there are differences between flower companies certified in the scheme Florverde Sustainable Flowers (FSF) and those that are not, in their social and environmental practices within the framework of social responsibility (SR). Based on RS practices included in FSF, an opinion survey was applied to managers and interviews were conducted with workers. The identification of differences between certified and non-certified companies allowed establishing the effects of FSF certification on the companies studied. Differences were found in the implementation of SR practices for 41 of the 74 variables analyzed in the environmental categories, labor practices and consumer affairs. The benefits of FSF certification are analyzed from two perspectives. From the internal perspective, FSF contributes to produce with a sustainable approach, to organize the internal processes of the companies and to comply with applicable legislation. From the external perspective, FSF improves the corporate image to the market, to workers and to the community. For these reasons, the flowers companies have in the FSF certification scheme a valuable tool to apply the core subjects and issues of $S R$ in its operations.

Keywords: Florverde, flowers, social responsibility, sustainability, social and environmental certification.

\section{INTRODUCCIÓN}

La floricultura colombiana mantiene una importante presencia en el comercio internacional desde hace varias décadas, lo que la ha llevado a convertirse en una cadena productiva significativa en términos económicos, sociales y ambientales. De acuerdo con la Asociación Colombiana de Exportadores de Flores (2012), la floricultura es el primer renglón de exportaciones agrícolas no tradicionales de Colombia, y genera en cerca de 6.500 hectáreas, 130.000 empleos formales directos e indirectos en 60 municipios.

La industria de las flores en el mundo es un ejemplo particularmente interesante de la aparición y proliferación de normas que buscan regular las condiciones sociales y ambientales de producción. El Centro para la promoción de las importaciones de los países en desarrollo (CBI) de los Países Bajos (2013) afirma que la Responsabilidad Social Empresarial (RSE) llegará a ser esencial dado que los supermercados han creado una creciente demanda de flores producidas de forma responsable.

La palabra responsabilidad indica la acción de responder por los resultados de las propias decisiones y acciones; la palabra social recuerda que esas decisiones y acciones afectan a otros (Herrera y Abreu, 2008). De acuerdo con el Centro Colombiano de Responsabilidad Empresarial (2005), la responsabilidad social se manifiesta cuando existe un conjunto sistemático de políticas, programas y prácticas coordinadas e integradas a la operación del negocio que soportan el proceso de toma de decisiones frente a cada una de las relaciones que la empresa mantiene, de manera coherente y con unos principios básicos de gestión.

Según Riisgaard (2009), en los últimos años los productores de flores en países en vías de desarrollo como Kenia y Colombia han sido blancos de críticas por parte de organizaciones en países de Europa y América del 
Norte, debido a los efectos de la producción de flores en las condiciones ambientales y sociales manejadas. En Kenia, por ejemplo, se han realizado algunos estudios sobre los impactos sociales y ambientales de la producción de flores. Algunas de estas investigaciones muestran las malas condiciones laborales de los trabajadores en las plantaciones de flores y la generación de impactos ambientales negativos (Riddselius, 2011).

Ante este panorama, el sector floricultor colombiano agremiado en Asocolflores creó en 1996 su propia iniciativa para estimular el mejoramiento continuo de las condiciones sociales y ambientales en las empresas floricultoras, a través del programa Florverde. El programa surgió en una década y unas circunstancias en que se cuestionaba el impacto que tenía la floricultura, así como otras actividades productivas en el ambiente y en la sociedad (Isaza, 2007). Esta iniciativa se constituyó en una de las estrategias del sector para lograr una floricultura competitiva, sostenible y con responsabilidad social, y sentó las bases de lo que hoy es el esquema de certificación Florverde Sustainable Flowers o FSF.

El estándar Florverde Sustainable Flowers consta de 190 requisitos agrupados en 15 capítulos: sistema de gestión, derechos laborales, formación y entrenamiento de los trabajadores, bienestar, gestión de la seguridad y salud en el trabajo, uso racional del agua, fertilización y manejo de suelos y sustratos, manejo integrado de plagas, manejo de residuos, manejo de la finca, el paisaje y la biodiversidad, eficiencia energética y mantenimiento de equipos y máquinas, origen del material vegetal, cuidado del producto en poscosecha, trazabilidad y registros, estado y uso de la marca de conformidad FSF (Asocolflores, 2013).

Después de realizar una revisión de literatura sobre el tema y de consultar los artículos publicados en los últimos diez años en las bases de datos ScienceDirect y Redalyc, únicamente se encontraron 16 artículos que mencionan la palabra clave Florverde. Esto confirmó que después de 18 años de su creación, no se ha desarrollado una investigación específica sobre el esquema de certificación Florverde Sustainable Flowers (FSF) que permita determinar si su aplicación ha contribuido a la mejora de las prácticas sociales y ambientales de las empresas, desde la perspectiva del desarrollo sostenible y la responsabilidad social.

No obstante, existen algunos estudios específicos sobre FSF que han evaluado las prácticas sociales, como los estudios de Korovkin y San Miguel (2007), Valero y Camacho (2006) o aspectos ambientales como el estudio Copete (2012) sobre el uso del agua. Así mismo, se han realizado trabajos de tesis de maestría sobre la importancia en la implementación de sellos socioambientales como la investigación de Martínez y Gómez (2013), y sobre la prospectiva del sector floricultor colombiano como el trabajo de Sierra y Blanco (2013), que muestran la relevancia de la RSE en el futuro de la industria de flores en el país.

Esta investigación pretendió determinar si la certificación FSF como parte de la estrategia de responsabilidad social del sector ha sido una herramienta eficaz para mejorar las prácticas sociales y ambientales de las empresas certificadas en comparación con las empresas no certificadas. También se buscó establecer los beneficios de la implementación y certificación de FSF y aportar recomendaciones que permitan orientar la toma de decisiones con respecto a su futuro como parte de las estrategias de responsabilidad social del sector floricultor.

\section{METODOLOGÍA}

Evaluar los efectos de Florverde Sustainable Flowers es una tarea compleja y podrían usarse varios enfoques para hacerlo, sin embargo, como se mencionó en la revisión de literatura, no existen estudios previos 
al respecto. Por estas razones se decidió realizar una investigación no experimental transversal mediante encuestas de opinión, ya que el propósito del estudio consistió en describir las variables de responsabilidad social y analizar su incidencia e interrelación. De acuerdo con Cubbage et al. (2010), en su estudio Impacts of forest management certification in Argentina and Chile, en el que se revisó la literatura existente sobre evaluaciones de impacto de la certificación forestal, este enfoque ha sido utilizado en múltiples evaluaciones de impacto de esquemas de certificación como el Forest Stewardship Council (o FSC, por sus siglas en inglés).

De acuerdo con lo anterior, se abordó una metodología multimétodo, que involucra la identificación de las prácticas de responsabilidad social incorporadas en el esquema FSF, la evaluación de las opiniones de los directivos de las empresas mediante una encuesta de opinión con respecto a las prácticas de responsabilidad social implementadas en las empresas certificadas y no certificadas y la realización de entrevistas a trabajadores en sus sitios de trabajo.

En la primera parte de la investigación se realizó un análisis de contenido de la norma Florverde Sustainable Flowers (FSF) y de la norma ISO 26000, esto con el propósito de determinar la correspondencia entre las materias fundamentales y asuntos de responsabilidad social de la norma ISO 26000 y los requisitos de la certificación FSF. Este análisis permitió establecer los asuntos de responsabilidad social incluidos en la certificación FSF y que se constituyeron en las variables de responsabilidad social de interés para la investigación.

Con base en la información anterior, se elaboró un instrumento que fue aplicado a una muestra de empresas floricultoras certificadas y no certificadas en FSF. El instrumento fue sometido a una validación previa por parte de expertos para corregir potenciales desviaciones. El instrumento diseñado estuvo compuesto por 4 preguntas de carácter general sobre las empresas, 8 preguntas de selección múltiple sobre aspectos de interés sobre la certificación FSF y las 74 variables de responsabilidad social identificadas, las cuales se evaluaron mediante una escala de Likert de 4 a 1 en la que 4 fue "totalmente de acuerdo", 3 fue "parcialmente de acuerdo", 2 fue "parcialmente en desacuerdo" y 1 fue "totalmente en desacuerdo".

$\mathrm{El}$ instrumento validado fue enviado por correo electrónico a las 240 empresas afiliadas a Asocolflores para ser respondido por sus directivos. Se obtuvo un total de 42 casos válidos que incluyeron 23 empresas certificadas FSF y 19 no certificadas FSF, las cuales corresponden a la muestra final de la investigación. $\mathrm{La}$ tasa de respuesta efectiva fue del $17.5 \%$. El proceso de recolección de datos se llevó a cabo entre agosto y noviembre de 2015.

El análisis de datos se realizó combinando un primer análisis descriptivo y un análisis exploratorio posterior de las 74 variables de responsabilidad social identificadas, que permitiera obtener posibles relaciones estadísticamente significativas entre algunas de ellas. Dada la naturaleza ordinal de escala utilizada en cada una de las variables analizadas, se empleó la prueba Kruskal-Wallis para corroborar si los valores alcanzados para cada variable se deben al hecho de que la empresa se encuentre certificada en FSF o no.

Para el análisis multivariado de las 74 variables se aplicaron dos técnicas de análisis: el análisis de correspondencia múltiple y el análisis de clúster. El análisis de correspondencia múltiple es una técnica de interdependencia que permite resumir una gran cantidad de información en un número reducido de dimensiones o factores, así como crear mapas perceptuales basados en la asociación entre casos y un conjunto de características descriptivas. El análisis de clúster es relevante 
en la investigación dado que con este análisis se buscó establecer si las variables analizadas permiten discriminar la muestra en grupos y si la certificación FSF es una característica que define a los grupos identificados.

Con la intención de dar contexto y facilitar el análisis de los resultados cuantitativos, se realizaron entrevistas en profundidad a trabajadores pertenecientes a 4 empresas de flores, 2 empresas certificadas y 2 no certificadas FSF. Las entrevistas en profundidad a los trabajadores se basaron en un instrumento previamente desarrollado y utilizado por Riddselius (2011) en su investigación de maestría Certification process of international standards in the Kenyan cut flower industry. A este instrumento se le realizó una adaptación lingüística debido a que fue desarrollado en el idioma inglés. Posteriormente se organizó según las categorías de análisis definidas y fue revisado por una experta en el esquema de certificación FSF antes de su aplicación. Las entrevistas fueron efectuadas a grupos de entre 7 y 9 trabajadores por finca. El tiempo de realización de cada entrevista osciló entre treinta y cinco minutos a una hora.

\section{RESULTADOS Y DISCUSIÓN}

\section{Identificación de prácticas de responsabilidad social en FSF}

Para definir el alcance del esquema FSF en términos de responsabilidad social, como primera medida se identificaron las materias fundamentales y los asuntos de responsabilidad social propuestos en la norma ISO 26000 que se encontraban incluidos en la norma FSF. Después de analizar el contenido de ambos documentos, se pudo establecer la relación entre los requisitos de la norma FSF con 18 de los 37 asuntos cubiertos por la norma ISO 26000, y se encontró que 74 acciones y expectativas definidas en la norma ISO 26000 para las materias fundamentales y los asuntos de responsabilidad social tenían convergencia con algún requisito de FSF.

\section{Implementación de prácticas de responsabilidad social en las empresas certificadas FSF y no certificadas}

En términos generales, las empresas participantes en el estudio se manifestaron totalmente de acuerdo frente a la mayor parte de los ítems evaluados, lo que quiere decir que han implementado en su organización alguna práctica relacionada con la variable analizada. Así, de las 3.108 respuestas recibidas, $2.021(65.0 \%)$ corresponden a la categoría "totalmente de acuerdo"; 791 (25.5\%) a la categoría "parcialmente de acuerdo"; 191 (6.1\%) a la categoría "parcialmente en desacuerdo"; y tan solo 105 (3.4\%) a la categoría "totalmente en desacuerdo".

Con relación a los 74 ítems de responsabilidad social que se constituyeron en las variables de este estudio, cabe mencionar que se presentaron diferencias estadísticas para 41 variables, las cuales se encuentran asociadas a las distintas materias y asuntos de responsabilidad social, pero principalmente a las categorías de medio ambiente (19 variables), prácticas laborales (10 variables) y asuntos de consumidores (5 variables). Cabe mencionar que en la materia fundamental de derechos humanos no se presentaron diferencias en ninguna variable. De hecho, hubo variables como "la organización no practica el trabajo forzoso u obligatorio" y "la organización no contrata menores de edad bajo ninguna modalidad", en las cuales el $100 \%$ de la muestra de empresas certificadas y empresas no certificadas FSF declararon que han implementado prácticas para garantizar su cumplimiento.

El análisis de clúster realizado dio como primer resultado un dendograma en el cual se puede observar la jerarquía del agrupamiento de los casos con 
respecto a las 74 variables del instrumento. Sin embargo, en este caso, el agrupamiento por medio del dendograma no permitió determinar con claridad a qué distancia se encuentran las agrupaciones realizadas. Dado que no se pudo determinar claramente cuántos clústeres se formaron, se utilizó la técnica de curvas de nivel indexadas y por medio de esta técnica se pudo deducir que es pertinente formar 4 clústeres homogéneos dentro de ellos, pero heterogéneos o diferentes entre ellos.
Una vez definidos los 4 grupos, fue necesario saber cuáles son las variables y las categorías de estas más influyentes en cada uno, las cuales son las que van a permitir describirlos de manera acertada. Con base en el análisis de correspondencia múltiple, se definieron dos factores con el fin de elaborar un mapa perceptual. Esto permitió ubicar espacialmente a las empresas e identificarlas de acuerdo con el grupo al que pertenecen. La figura 1 muestra la agrupación de casos realizada en los 4 clústeres definidos.

Figoura 1. Mapa perceptual de dos factores - análisis de correspondencia múltiple.

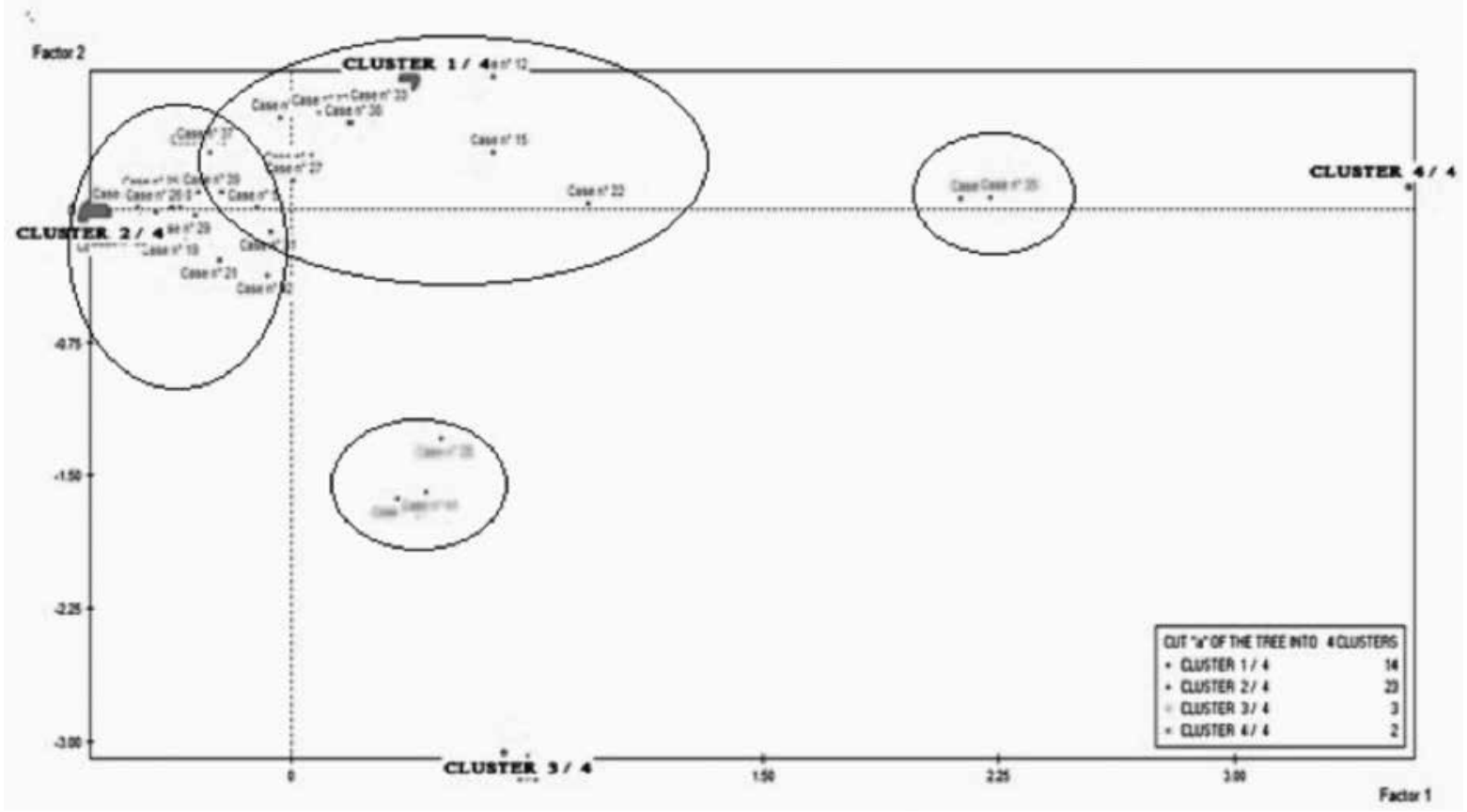

Fuente: elaboración propia.

Figura 2. Composición de los clústeres identificados.

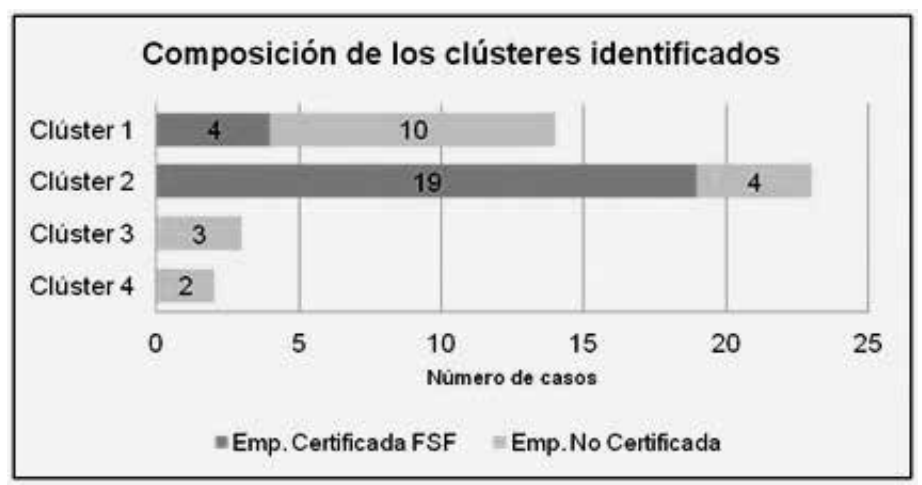

Fuente: elaboración propia. 
En el primer grupo se encuentran 14 empresas, en el segundo grupo se encuentran 23 empresas, convirtiéndolo en el grupo más grande, por lo tanto, el que representa más similitudes; $\mathrm{y}$, por último, los grupos tercero y cuarto recogen solo 5 empresas.

Como se aprecia en la figura 2, la composición de cada clúster reafirma las diferencias existentes entre las empresas certificadas FSF y las empresas no certificadas. Las empresas certificadas FSF se encuentran, principalmente, en el clúster 2, lo que constituye el 82.6 $\%$ de este grupo, por lo cual se denominó "clúster de empresas certificadas". El restante $17.4 \%$ de empresas certificadas se ubicaron en el clúster 1. Por otro lado, las empresas no certificadas se ubicaron, básicamente, en el clúster 1 (71.4\%), por lo cual se nombró este grupo como "clúster de empresas no certificadas tipo".
Los clústeres 3 y 4 no son muy representativos y están compuestos por las empresas no certificadas que tuvieron las evaluaciones más bajas en el instrumento. $\mathrm{Si}$ bien existen diferencias entre las empresas que componen cada uno de estos dos clústeres, por su tamaño tan pequeño no se analizaron en detalle y se denotaron como "clústeres de empresas no certificadas atíicicas".

Una vez se identificaron las empresas que pertenecen a cada clúster, fue necesario saber cuáles eran las variables que más discriminaban entre los clústeres y que generaban similitudes entre las empresas de cada clúster; por ello, fue necesario examinar una a una cada variable. En la tabla 1 se presentan las variables significativas $(\mathrm{p}<0,05)$ que más caracterizan a los clústeres 1 y 2, así como la respuesta predominante para cada uno.

Tabla 1. Variables significativas que caracterizan a los clústeres identificados.

\begin{tabular}{|c|c|c|c|c|c|}
\hline \# & Categoría / Subcategoría & Variable * & $\begin{array}{l}\text { Parcialmente en } \\
\text { desacuerdo }\end{array}$ & $\begin{array}{l}\text { Parcialmente } \\
\text { de acuerdo }\end{array}$ & $\begin{array}{l}\text { Totalmente } \\
\text { de acuerdo }\end{array}$ \\
\hline 15 & $\begin{array}{l}\text { Gobierno corporativo } \\
\text { Direccionamiento estratégico }\end{array}$ & $\begin{array}{l}\text { La organización cuenta con mecanismos } \\
\text { sociolaborales para promover la participación de los } \\
\text { trabajadores. }\end{array}$ & & Clúster 1 & Clúster 2 \\
\hline 27 & $\begin{array}{l}\text { Prácticas laborales } \\
\text { A1. Trabajo y relaciones laborales }\end{array}$ & $\begin{array}{l}\text { La organización da información oportuna a los } \\
\text { trabajadores cuando se presentan cambios que } \\
\text { pueden afectar su estabilidad laboral. }\end{array}$ & Clúster 1 & & Clúster 2 \\
\hline 29 & $\begin{array}{l}\text { Prácticas laborales } \\
\text { A1. Trabajo y relaciones laborales }\end{array}$ & $\begin{array}{l}\text { La organización cuenta con mecanismos que } \\
\text { permiten asegurar la privacidad y confidencialidad } \\
\text { de los datos de los trabajadores. }\end{array}$ & & Clúster 1 & Clúster 2 \\
\hline 30 & $\begin{array}{l}\text { Prácticas laborales } \\
\text { A1. Trabajo y relaciones laborales }\end{array}$ & $\begin{array}{l}\text { La organización promueve y controla prácticas } \\
\text { laborales justas de sus contratistas y proveedores. }\end{array}$ & & Clúster 1 & Clúster 2 \\
\hline 38 & $\begin{array}{l}\text { Prácticas laborales } \\
\text { A4. Salud y seguridad ocupacional }\end{array}$ & $\begin{array}{l}\text { La organización ha comunicado a sus trabajadores y } \\
\text { cumple su política salud y seguridad en el trabajo. }\end{array}$ & & Clúster 1 & Clúster 2 \\
\hline 43 & $\begin{array}{l}\text { Prácticas laborales } \\
\text { A4. Salud y seguridad ocupacional }\end{array}$ & $\begin{array}{l}\text { La organización registra, reporta e investiga } \\
\text { todos los accidentes de trabajo y los incidentes } \\
\text { en materia de salud y seguridad, con el objeto de } \\
\text { minimizarlos o eliminarlos. }\end{array}$ & Clúster 1 & & Clúster 2 \\
\hline 61 & $\begin{array}{l}\text { Medio ambiente } \\
\text { A2. Uso sostenible de los recursos }\end{array}$ & $\begin{array}{l}\text { La organización identifica, mide y registra el } \\
\text { consumo de los recursos utilizados (agua, energía, } \\
\text { combustibles, otros). }\end{array}$ & & Clúster 1 & Clúster 2 \\
\hline 62 & $\begin{array}{l}\text { Medio ambiente } \\
\text { A2. Uso sostenible de los recursos }\end{array}$ & $\begin{array}{l}\text { La organización identifica e implementa medidas } \\
\text { para la optimización de los recursos utilizados. }\end{array}$ & & Clúster 1 & Clúster 2 \\
\hline
\end{tabular}




\begin{tabular}{|c|c|c|c|c|c|}
\hline$\#$ & Categoría / Subcategoría & Variable* & $\begin{array}{l}\text { Parcialmente en } \\
\text { desacuerdo }\end{array}$ & $\begin{array}{l}\text { Parcialmente } \\
\text { de acuerdo }\end{array}$ & $\begin{array}{l}\text { Totalmente } \\
\text { de acuerdo }\end{array}$ \\
\hline 64 & $\begin{array}{l}\text { Medio ambiente } \\
\text { A2. Uso sostenible de los recursos }\end{array}$ & $\begin{array}{l}\text { La organización cuenta con programas de ahorro y } \\
\text { uso eficiente del agua y de la energía. }\end{array}$ & & Clúster 1 & Clúster 2 \\
\hline 65 & $\begin{array}{l}\text { Medio ambiente } \\
\text { A2. Uso sostenible de los recursos }\end{array}$ & $\begin{array}{l}\text { La organización utiliza materiales reciclados para el } \\
\text { desarrollo de sus actividades. }\end{array}$ & & Clúster 1 & Clúster 2 \\
\hline 66 & $\begin{array}{l}\text { Medio ambiente } \\
\text { A2. Uso sostenible de los recursos }\end{array}$ & $\begin{array}{l}\text { La organización promueve y trabaja estrategias de } \\
\text { consumo sostenible. }\end{array}$ & & Clúster 1 & Clúster 2 \\
\hline 71 & $\begin{array}{l}\text { Medio ambiente } \\
\text { A4. Protección del medio } \\
\text { ambiente, la biodiversidad y } \\
\text { restauración de hábitats }\end{array}$ & $\begin{array}{l}\text { La organización identifica impactos negativos } \\
\text { potenciales sobre la biodiversidad y toma medidas } \\
\text { para eliminar, minimizar o compensar dichos } \\
\text { impactos. }\end{array}$ & & Clúster 1 & Clúster 2 \\
\hline 78 & $\begin{array}{l}\text { Asuntos de consumidores } \\
\text { A2. Protección de la salud y } \\
\text { seguridad de los consumidores }\end{array}$ & $\begin{array}{l}\text { La organización suministra productos o servicios } \\
\text { que al ser usados y almacenados en condiciones } \\
\text { normales son seguros para los usuarios y para el } \\
\text { medio ambiente. }\end{array}$ & & Clúster 1 & Clúster 2 \\
\hline 79 & $\begin{array}{l}\text { Asuntos de consumidores } \\
\text { A2. Protección de la salud y } \\
\text { seguridad de los consumidores }\end{array}$ & $\begin{array}{l}\text { La organización cuenta con herramientas que le } \\
\text { permitan controlar aspectos relacionados con la } \\
\text { calidad de los productos o servicios frente a la } \\
\text { comercialización y consumo de productos que } \\
\text { puedan ir en contra de la salud y seguridad de los } \\
\text { usuarios. }\end{array}$ & & Clúster 1 & Clúster 2 \\
\hline
\end{tabular}

Fuente: elaboración propia.

$*(\mathrm{p}<0.05)$

En total, fueron significativas 14 variables del estudio en el agrupamiento para ambos clústeres. Las categorías de análisis que más generaron diferencias en el análisis de clúster fueron nuevamente las de medio ambiente, que registró 6 variables significativas, y la de prácticas laborales, que tuvo 5 variables significativas. Cabe señalar que las variables de la categoría de medio ambiente identificadas a través del análisis de clúster (variables 61, 62, 64, 65, 66 y 71) y la variable 79 de la categoría de asuntos de consumidores son consistentes con las diferencias estimadas en el análisis inicial de la encuesta mediante la prueba de Kruskal-Wallis.

\section{Análisis de las entrevistas a trabajadores de empresas certificadas y no certificadas}

Centrarse en este grupo de interés permitió obtener una comprensión de los efectos de FSF en las prácticas laborales y obtener resultados comparables entre las empresas certificadas y no certificadas. Las entrevistas permitieron tener un acercamiento directo a las prácticas de responsabilidad social de las empresas en el sitio de trabajo. Vale la pena mencionar que, después del análisis de contenido de las entrevistas, no se identificaron mayores diferencias en cuanto a las materias fundamentales evaluadas entre las empresas certificadas FSF y las no certificadas. La información recopilada en las entrevistas confirmó los resultados obtenidos mediante las encuestas frente a las prácticas sociales implementadas en las empresas con relación a las materias fundamentales y la mayor parte de asuntos de responsabilidad social sobre prácticas laborales y derechos humanos, no así para los asuntos de derechos civiles y políticos y diálogo social, en los cuales, contrario a lo expresado por los directivos de las empresas en las encuestas frente a la libertad de asociación y negociación colectiva, lo manifestado durante las entrevistas a los trabajadores refleja que este tema sigue siendo polémico en el sector floricultor y 
presenta resistencia por parte de las empresas. Ante esta situación, FSF no ha logrado generar ningún impacto al interior de las empresas certificadas.

Este comportamiento coincide con las críticas realizadas por Korovkin y Sanmiguel (2007) en cuanto a que es poco lo que puede aportar FSF al ejercicio de los derechos laborales básicos. También es concordante con la posición de Herrera et al. (2004) en cuanto a que Florverde (hoy FSF) no contempla aspectos importantes en la esfera laboral, como el derecho a la asociación y a la negociación colectiva.

\section{Principales beneficios de la certificación FSF}

Los resultados encontrados acerca de los beneficios de la certificación FSF son coherentes con los hallazgos encontrados por Martínez y Gómez (2013) en su estudio. Si se comparan los beneficios que genera la certificación para las empresas según esta investigación con los reportados en el estudio citado, se puede afirmar que, a juicio de los directivos, los beneficios de la certificación FSF se pueden analizar desde dos perspectivas: una interna y otra externa.

Desde la perspectiva interna, los resultados muestran que la certificación FSF contribuye fundamentalmente a producir con un enfoque sostenible, organizar los procesos internos de la empresa y cumplir con la legislación aplicable. En este sentido, de acuerdo con Martínez y Gómez (2013), los gerentes de empresas certificadas manifiestan que se ha generado conciencia de producir y consumir productos más "naturales", motivo por el cual la implementación de sellos ambientales como FSF ha llevado a las empresas a establecer controles en sus operaciones, a propiciar la preservación del medio ambiente y a minimizar los impactos ambientales que puedan ser ocasionados por su operación.

Desde la perspectiva externa, los directivos de las empresas consideran que con la certificación FSF la imagen de las empresas mejora ante el mercado al ofrecer un producto sostenible, así como mejora ante los trabajadores y ante la comunidad. Martínez y Gómez (2013) concuerdan en señalar que, para los directivos de las empresas, el hecho de que en el país se tengan empresas con sellos socioambientales ayuda de manera notable a tener un reconocimiento internacional que lo hace destacarse de los demás países que exportan flores.

No obstante, a pesar de que la mayoría de los directivos de las empresas estima que con la certificación FSF la imagen de las flores colombianas mejora al ofrecer al mercado un producto sostenible, solo el $9.5 \%$ consideró la apertura de nuevos mercados como una razón para certificarse en FSF. Lo anterior plantea un reto clave para el esquema FSF en términos de mejorar su posicionamiento en el mercado y lograr el reconocimiento de la certificación por parte de los miembros de la cadena de valor en el ámbito internacional.

\section{CONCLUSIONES}

El propósito principal de esta investigación era determinar si existían diferencias entre las empresas de flores certificadas en Florverde Sustainable Flowers y las que no lo están en cuanto a sus prácticas sociales y ambientales en el marco de la responsabilidad social. La identificación de estas diferencias permitió establecer los efectos que en términos sociales y ambientales ha generado la implementación y certificación del esquema FSF en las empresas floricultoras estudiadas.

Se encontraron diferencias estadísticas según la prueba de Kruskal-Wallis para 41 de las 74 variables analizadas individualmente. Esto indica que es en dichas categorías, y particularmente en las variables identificadas, en las que se presenta el impacto del esquema de certificación FSF al interior de las empresas certificadas en términos de responsabilidad social, ya que en todas 
las diferencias identificadas las empresas certificadas tuvieron un valor más alto en la escala de evaluación que las empresas no certificadas. Los resultados encontrados en el análisis multivariado mediante el análisis de clúster y el análisis de correspondencia múltiple confirmaron que sí existen diferencias estadísticas en la implementación de prácticas de responsabilidad social por parte de las empresas certificadas FSF y no certificadas incluidas en la investigación, por lo tanto, se concluye que la hipótesis planteada, "existen diferencias estadísticas en cuanto a las prácticas sociales y ambientales entre las empresas certificadas FSF y las empresas no certificadas", se acepta en el caso de las 41 variables analizadas de forma independiente y de las 14 variables analizadas de manera simultánea.

Las entrevistas a trabajadores permitieron tener un acercamiento directo a las prácticas de responsabilidad social de las empresas en el sitio de trabajo y confirmar la mayoría de las opiniones expresadas en las encuestas por parte de los directivos de las empresas. Sin embargo, cabe destacar que con relación a las variables "la organización permite y respeta el derecho de libertad de asociación y el derecho a la negociación colectiva" y "la organización no obstruye, amenaza o toma represalias hacia los trabajadores que busquen formar sus propias organizaciones o unirse a ellas y negociar colectivamente", en las entrevistas a trabajadores se evidenciaron dos cosas importantes. La primera es la percepción negativa que hay en las empresas de flores acerca de los temas relativos a la libertad de asociación, la negociación colectiva y los sindicatos; la segunda es el desconocimiento que tienen los trabajadores frente al tema. Esto permite concluir que el esquema FSF no ha logrado generar ningún impacto al respecto en las empresas certificadas.

En cuanto a los beneficios de la certificación FSF, los resultados permiten afirmar que a juicio de los directivos de las empresas de flores, los beneficios de la certificación FSF se pueden analizar desde dos perspectivas, una interna y otra externa. Desde la perspectiva interna, el esquema FSF contribuye a producir con un enfoque sostenible, a organizar los procesos internos de la empresa y a cumplir con la legislación aplicable. Desde la perspectiva externa, los directivos de las empresas consideran que con la certificación FSF la imagen de las empresas mejora ante el mercado al ofrecer un producto sostenible, así como mejora ante los trabajadores y ante la comunidad.

Dada la trayectoria y experiencia acumulada por el esquema Florverde Sustainable Flowers a lo largo de los años, es decisivo que esta iniciativa pueda continuar brindando su apoyo a las empresas, para lo cual debe alinearse con los retos que afronta el sector floricultor y con las necesidades y expectativas de sus partes interesadas. Por lo anterior se recomienda incluir en la norma FSF las materias fundamentales y los asuntos de responsabilidad social no contemplados actualmente, teniendo en cuenta el contexto actual de la producción de flores, así como la prospectiva del sector, con el fin de identificar aquellos asuntos que sean más pertinentes para las organizaciones y que puedan contribuir a enfrentar los desafíos presentes y futuros, y así garantizar la sostenibilidad de esta actividad, de enorme importancia para el país. Particularmente se recomienda la inclusión de los siguientes requisitos en la norma FSF:

Derechos humanos. Una política de derechos humanos que incluya el no consentir abusos tales como actos de discriminación laboral, actos de desplazamiento forzado, alianzas formales o informales o relaciones contractuales con contrapartes que cometan abusos de los derechos humanos.

Prácticas laborales. Demostrar que las organizaciones no obstruyen a los trabajadores que busquen formar sus propias organizaciones o unirse a ellas para negociar colectivamente, por ejemplo, despidiéndolos o 
discriminándolos, a través de represalias que creen un ambiente de intimidación o miedo.

Medio ambiente. La identificación de los aspectos e impactos ambientales de las actividades. En cuanto a la mitigación del cambio climático es importante la exigencia de implementar programas para mejorar la eficiencia y la eficacia en el uso de combustibles.

Prácticas justas de operación. La identificación de riesgos de corrupción y la implementación de políticas y prácticas que la combatan. También se debe propiciar la integración de criterios éticos, sociales, ambientales, de salud y seguridad en las políticas de compra, de venta y de contratación de las empresas.

Participación activa y desarrollo de la comunidad. La creación de mecanismos de participación en iniciativas locales que pueda tener la comunidad de su entorno y el análisis del impacto de las decisiones de la empresa sobre la creación o mantenimiento de los empleos.

Para mejorar la eficacia del esquema de certificación FSF como herramienta para el cumplimiento legal de las empresas de flores, se recomienda generar un anexo en el cual se relacionen los requisitos de FSF con los referentes legales pertinentes. Esto facilitará a las empresas el entendimiento de la norma y sus fundamentos en la legislación, y orientará a las organizaciones hacia el cumplimiento de los requisitos legales en sus operaciones.

Con el propósito de aumentar la transparencia del esquema FSF y su involucramiento con las partes interesadas de cara a su reconocimiento internacional, se recomienda incluir en el comité que elabora y revisa los documentos normativos del esquema FSF a un mayor número de voces interesadas. Para este objetivo, puede ser útil considerar el mapa de grupos de interés presentado en el reporte de sostenibilidad de Asocolflores.

Las empresas del sector floricultor tienen en el esquema FSF una valiosa herramienta para aplicar los principios, las materias fundamentales y los asuntos de responsabilidad social en sus operaciones. Sin embargo, la responsabilidad social de las empresas de flores no depende únicamente de la decisión de implementar y certificarse en la norma FSF. Es necesario involucrar varios factores para que las empresas de flores colombianas puedan llegar a considerarse como "socialmente responsables" por parte de sus grupos de interés. Entre los factores clave en este proceso se encuentran el compromiso socioambiental real de la alta dirección, la mayor participación de los trabajadores en los procesos de implementación de las prácticas de responsabilidad social, así como la identificación y vinculación de las partes interesadas y sus expectativas.

Para garantizar que estos procesos se mantengan en el tiempo, es igualmente necesario que el cumplimiento con estas prácticas sea reconocido por los actores del mercado a través de la preferencia a la hora de tomar las decisiones de compra.

Se reconoce la necesidad de estudiar la contribución de la responsabilidad social a la sostenibilidad de las organizaciones del sector floricultor de una manera más profunda, dado que dentro de esta investigación se asumió como premisa básica que el objetivo de la responsabilidad social es contribuir al desarrollo sostenible de las organizaciones, pero no se llegó a identificar ni la magnitud de esta contribución ni en qué dimensiones del desarrollo sostenible se logra.

Complementando la recomendación anterior, y para dar continuidad a este trabajo en el área de la responsabilidad social, se propone el desarrollo de un estudio de identificación y caracterización de prácticas de responsabilidad social en las empresas de flores que no se limite a las prácticas que fueron identificadas en el contenido de la norma FSF, marco normativo de esta investigación. Se debe considerar que algunas empresas del sector, con apoyo de Asocolflores y de otras entidades, vienen avanzado en la implementación de 
acciones que van más allá de las prácticas recomendadas por la norma FSF; sin embargo, esto no está documentado en el presente estudio.

\section{REFERENCIAS}

Asociación Colombiana de Exportadores de Flores. (2012). Informe de actividades 2012: floricultura sostenible con responsabilidad social. Bogotá: Asocolflores.

Asociación Colombiana de Exportadores de Flores. (2013). Estándar Florverde para la producción sostenible de flores y ornamentales. Bogotá: Asocolflores.

Centro Colombiano de Responsabilidad Empresarial. (2005). ¿Quées la responsabilidad social empresarial? Bogotá. Recuperado de http://www.icesi.edu.co/ blogs/paoladministradora/files/2012/06/que-esrse.pdf

Copete, N. (2012). Evaluación formativa de la gestión ambiental del programa finca Florverde en relación con el manejo de agua en la sabana de Bogotá. (Tesis de maestría en Gestión Ambiental). Bogotá: Universidad Javeriana. Facultad de Estudios Ambientales y Rurales.

Cubbage, F., Díaz, D., Yapura, P., y Dube, F. (2010). Impacts of forest management certification in Argentina and Chile. Forest Policy and Economics, (12), 497-504.

Herrera, B., Puyo, L., Londoño, O., Soto, N., y Castaño, J. (2004). Frutas y flores de exportación: las condiciones laborales de las trabajadoras en Chile y Colombia. Santiago de Chile: Oxfam.

Herrera, J., y Abreu, J. (2008). Cómo gestionar la responsabilidad social en las Pymes colombianas. International Journal of Good Conscience, 3(1), 395-425.
Isaza, J. (2007). FlorVerde® Diez años liderando cambios. Revista Asocolflores, (68), 11-20.

Korovkin, T., y Sanmiguel, O. (2007). Estándares de trabajo e iniciativas no estatales en las industrias florícolas de Colombia y Ecuador. Revista Iconos de Ciencias Sociales, (29),15-30.

Martínez, A., y Gómez, L. (2013). Evaluación de la importancia en la implementación de sellos socio ambientales (Rainforest Alliance \& Florverde Sustainable Flowers) para los diferentes eslabones de la cadena del sector floricultor colombiano. (Tesis de maestría en Administración de Negocios). Bogotá: Universidad Sergio Arboleda.

Organización Internacional de Estandarización. (2010). Guía de Responsabilidad Social ISO 26000:2010. Geneve: ISO.

Países Bajos. Ministerio de Relaciones Exteriores. Centro para la Promoción de las Importaciones de los países en desarrollo. (2013). Mapa de Tendencias del CBI para Flores Cortadas. Recuperado de http://www.cbi.eu/

Riddselius, C. (2011). Certification process of international standards in the Kenyan cutflower industry. (Master thesis in Human Geography). Stockholm: Stockholm University. Department of Human Geography.

Riisgaard, L. (2009). Labour agency and private social standards in the cut flower industry. (PhD thesis). Copenhagen: Roskilde University, Department of Society and Globalization.

Sierra, M., y Blanco, L. (2013). Análisis prospectivo del sector floricultor colombiano al año 2022. (Tesis de maestría en Administración de Negocios). Bogotá: Universidad Sergio Arboleda.

Valero, E., y Camacho, K. (2006). El lado oscuro en las prácticas de responsabilidad social corporativa del sector floricultor. Revista Innovar, 16(27), 13-30. 